\title{
Correction to: Frequency domain approach for probabilistic flutter analysis using stochastic finite elements
}

\author{
Sandeep Kumar • Amit Kumar Onkar (D) Manjuprasad Maligappa
}

Published online: 8 January 2020

(C) Springer Nature B.V. 2020

\section{Correction to: Meccanica}

https://doi.org/10.1007/s11012-019-01061-9

Due to an unfortunate turn of events, this article was published with a erroneous version of Table 1. Please find in this document the correct version of Table 1 that should be regarded as the final version by the reader.
The original article can be found online at https:// doi.org/10.1007/s11012-019-01061-9.

S. Kumar · A. K. Onkar $(\bowtie) \cdot$ M. Maligappa Academy of Scientific and Innovative Research at CSIR National Aerospace Laboratories, HAL Airport Road, Kodihalli, Bengaluru 560017, India e-mail: aeroamit@nal.res.in

S. Kumar · A. K. Onkar · M. Maligappa Structural Technologies Division, National Aerospace Laboratories, HAL Airport Road, Kodihalli, Bengaluru 560017, India
Table 1 Properties of Goland wing [20]

\begin{tabular}{lll}
\hline Parameters & Description & Values \\
\hline$E I$ & Span-wise bending stiffness & $9.77 \times 10^{6} \mathrm{Nm}^{2}$ \\
$G J$ & Span-wise torsion stiffness & $0.988 \times 10^{6} \mathrm{Nm}^{2}$ \\
$m$ & Mass per unit span & $35.719 \mathrm{~kg} / \mathrm{m}$ \\
$x_{\alpha}$ & Dimensionless static & 0.33 \\
& $\quad$ unbalance & \\
$a$ & Elastic axis location & -0.2 \\
& $\quad$ parameter & $0.9144 \mathrm{~m}$ \\
$b$ & Semi-reference chord & $6.09 \mathrm{~m}$ \\
$l$ & Span & $6.5704 \mathrm{kgm} / \mathrm{m}$ \\
$I_{p}$ & Mass moment of inertia per & \\
& $\quad$ unit span & $1.225 \mathrm{~kg} / \mathrm{m}^{3}$ \\
\hline
\end{tabular}

Publisher's Note Springer Nature remains neutral with regard to jurisdictional claims in published maps and institutional affiliations. 